\title{
Gesundheitsbezogene Lebensqualität bei Patienten mit Heimbeatmung
}

\author{
W. Windisch ${ }^{1}$ \\ K. Freidel ${ }^{2}$ \\ H. Matthys ${ }^{1}$ \\ F. Petermann ${ }^{3}$
}

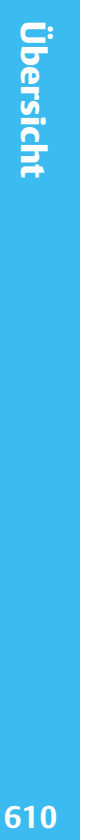

\author{
Health-Related Quality of Life (HRQL) in Patients Receiving Home Mechanical \\ Ventilation
}

\section{Zusammenfassung}

Neben der Lebensverlängerung hat die Lebensqualität eines Patienten in den letzten Jahren als neues Behandlungskriterium entscheidendes Gewicht bei der Beurteilung des medizinischen Therapieerfolges erhalten. Die gesundheitsbezogene Lebensqualität ist als psychologisches Konstrukt definiert, das in einem mehrdimensionalen Modell das subjektive Befinden eines Patienten auf vier minimal zu definierenden Grundsäulen charakterisiert, nämlich unter Berücksichtigung physischer, psychischer, sozialer und funktionaler Aspekte. Zur Messung der gesundheitsbezogenen Lebensqualität werden in erster Linie Fragebogen eingesetzt, die als krankheitsübergreifende Instrumente einen Vergleich zwischen verschiedenen Erkrankungsgruppen erlauben oder als krankheitsspezifische Instrumente auf spezielle Probleme einer definierten Erkrankung eingehen und so auf eine möglichst sensible Erfassung von Änderungen der gesundheitsbezogenen Lebensqualität, z. B. im Verlauf einer medizinischen Intervention, abzielen.

Die Heimbeatmung über eine Gesichtsmaske hat sich in den letzten Jahren als Behandlungsverfahren der chronischen ventilatorischen Insuffizienz etabliert. Während dabei ein Überlebensvorteil bei den meisten Patienten mit thorakal-restriktiven und neuromuskulären Erkrankungen als gesichert gilt, bleibt dies bei Patienten mit COPD weiter unklar. Ungeachtet dessen haben Patienten mit Heimbeatmung eine lange Krankheitsgeschichte über viele Jahre mit objektiv starken Beeinträchtigungen im Alltag. Darüber hinaus ist die Heimbeatmung eine zeit-

\section{Abstract}

Evaluation of health-related quality of life (HRQL) has become steadily more essential during the last two decades in research and health care practice in order to evaluate the human and financial costs and benefits of modern medical techniques. HRQL in its definition is based on different components of health including physical state, psychological well-being, social relations and functional capacities that are influenced by a persons experience, beliefs, expectations, and perceptions. For the purpose of assessment of HRQL several instruments have been developed. Generic instruments are not specific to any particular disease and are therefore most commonly used for general survey research on health allowing comparisons between disease states. In contrast, disease-specific questionnaires which are necessary in order to focus on domains most relevant to a particular disease are thought to be more sensitive than generic instruments following therapeutic interventions.

Home mechanical ventilation (HMV) delivered noninvasively by a facial mask is a well established treatment for chronic hypercapnic respiratory failure. It is widely accepted that survival improves following institution of HMV in most patients with chest wall deformities or neuromuscular diseases while this is still controversially discussed in patients with COPD. However, patients receiving HMV usually have severe respiratory insufficiency with a past medical history of several years or decades, and suffer from end stage disease with objectively severe limitations of daily living. In addition, HMV is a time consuming and cost in-

Institutsangaben

${ }^{1}$ Abteilung Pneumologie, Universitätsklinik Freiburg (Ärztlicher Direktor: Prof. Dr. J. Müller-Quernheim)

${ }^{2}$ St. Josefs-Hospital Wiesbaden (Stabsstelle Qualitätsmanagement)

${ }^{3}$ Zentrum für Klinische Psychologie und Rehabilitation der Universität Bremen

(Direktor: Prof. Dr. F. Petermann)

Danksagung

Die Autoren bedanken sich herzlich bei Herrn Prof. Dr. med. C. P. Criée

für die Mithilfe bei der Erstellung des Manuskriptes.

Korrespondenzadiresse

Dr. med. W. Windisch · Abteilung Pneumologie, Universitätsklinik Freiburg ·

Killianstraße 5· 79106 Freiburg ·E-mail: windisch@med1.ukl.uni-freiburg.de

Bibliografie

Pneumologie 2002; 56: 610-620 @ Georg Thieme Verlag Stuttgart · New York · ISSN 0934-8387 
aufwendige und kostenintensive Therapie. Aus diesen Gründen sind insbesondere im letzten Jahrzehnt mehrere Untersuchungen zur gesundheitsbezogenen Lebensqualität bei Heimbeatmung durchgeführt worden.

Studien aus jüngster Zeit konnten unter Einsatz von krankheitsübergreifenden Fragebogen zeigen, dass die gesundheitsbezogene Lebensqualität bei Patienten mit Heimbeatmung verglichen mit Normalkollektiven eingeschränkt ist. Dabei zeigten sich Einschränkungen insbesondere in der körperlichen Gesundheit, nicht so sehr jedoch in der psychischen Gesundheit, was belegt, dass selbst stärkere körperliche Einschränkungen nicht notwendigerweise auch psychische Beeinträchtigungen nach sich ziehen müssen. Darüber hinaus sind die Einschränkungen nicht wesentlich stärker als bei Patienten mit anderen chronischen Erkrankungen, die nicht vom Respirator abhängig sind. Verbesserungen der gesundheitsbezogenen Lebensqualität durch die Heimbeatmung waren bei Patienten mit COPD nicht oder nur in geringem Maße zu erheben. Allerdings wird davon ausgegangen, dass Patienten mit restriktiven Erkrankungen mehr von der Heimbeatmung profitieren. Kürzlich wurden jedoch gut validierte Messinstrumente speziell für Patienten mit schwerer respiratorischer Insuffizienz entwickelt, bei denen eine höhere Änderungssensibilität bezüglich der gesundheitsbezogenen Lebensqualität postuliert wird. Solange allerdings die Ergebnisse prospektiver Studien mit diesen Instrumenten noch ausstehen, kann der Einfluss der Heimbeatmung auf die gesundheitsbezogene Lebensqualität nicht sicher beurteilt werden. tensive therapy. Therefore, several studies have been conducted in the last decade to evaluate HRQL in patients receiving HMV.

Recent studies using generic questionnaires have shown impairments in HRQL in patients receiving HMV compared to normals. This was primarily attributed to severe limitations in physical health, but not in mental health indicating that if severe physical limitation occurs in advanced respiratory disease this will not necessarily lead to mental limitation. In addition, limitations in HRQL in patients with HMV were not substantially higher than in patients with different chronic disease being not dependent on HMV. Improvements in HRQL following the institution of HMV were only mild or even insignificant in patients with COPD, but patients with restrictive ventilatory disorders are suspected to have more benefits. However, well validated disease-specific questionnaires which are designed to be more sensitive in the assessment of changes in HRQL than generic instruments have been introduced recently for patients with severe respiratory failure, but the influence of HMV to HRQL remains still unclear, since prospective studies using these questionnaires have yet not been finished.

\section{Einleitung}

Die Entwicklung der modernen Medizin hat in den jüngsten Jahrzehnten zu einer deutlichen Ausweitung der Therapiemöglichkeiten chronischer Krankheiten geführt. Im Zuge dieser Entwicklung konnte für eine Vielzahl dieser chronischen Krankheiten eine Lebensverlängerung erreicht werden, auch wenn das Grundleiden nicht kuriert werden kann. Dabei ist in zunehmendem Maße die Frage entstanden, ob die dazu gewonnene Lebenszeit auch lebenswert ist. Diese Frage nach der Lebensqualität eines Patienten muss auch bei Patienten mit chronischer respiratorischer Insuffizienz, die auf eine Heimbeatmungstherapie eingeleitet werden, gestellt werden.

Die vorliegende Übersichtsarbeit hat das Ziel, den gegenwärtigen Forschungsstand zum Thema „Lebensqualität bei Heimbeatmung“ zu skizzieren. Dabei wird gezeigt werden, dass die Frage nach der geeigneten Methodik der Erfassung von Lebensqualität in diesem Patientenkollektiv noch nicht hinreichend beantwortet ist, da die Entwicklung geeigneter Fragebogen aktuell noch Gegenstand wissenschaftlicher Bemühungen ist. Eine definitive Conclusio bezüglich der Lebensqualität bei Heimbeatmung kann somit nach aktueller Studienlage nicht hinreichend abgeleitet werden. Die vorliegende Übersichtsarbeit möchte daher eine Sensibilisierung für das Thema erreichen, insbesondere vor dem Hintergrund, dass die Zahl sowohl der Zentren, die das Therapieverfahren der Heimbeatmung anbieten, als auch der Patienten selbst, die auf eine Heimbeatmung eingeleitet werden, steigend ist. Dabei soll gezeigt werden, wie wichtig neue Forschungsvorhaben für die Erschließung der Frage nach der Lebensqualität in dieser Patientengruppe sind. Die vorliegende Übersichtsarbeit bietet somit eine Basis hierfür und hofft, die wissenschaftlichen Bemühungen um diesen Themenkomplex zu beleben.

\section{Heimbeatmung}

Seit den 60er Jahren und insbesondere seit Beginn der 80er Jahre hat die nichtinvasive Positivdruck-Beatmung (NPPV = noninvasive positive pressure ventilation) Einzug in die klinische Praxis zur Behandlung der respiratorischen Insuffizienz erhalten $[1,2]$. Als Beatmungszugang dient eine Gesichtsmaske, entweder als Nasenmaske (Abb.1) oder als Nasen-Mundmaske. Die NPPV hat sich initial bei Patienten mit chronischer respiratorischer Insuffizienz etabliert, wobei eine intermittierende Selbstbeatmung (ISB) als Dauertherapie nach stationärer Einleitung zu Hause durchgeführt wird (Heimbeatmung). Patienten mit COPD, neuromuskulären Erkrankungen oder thorakal-restriktiven Erkrankungen stellen das Hauptkollektiv für eine Heimbeatmung. Atemantriebsstörungen, das Adipositas-Hypoventilationssyndrom, Zwerchfellparesen, die Mukoviszidose sowie Bronchiektasen sind seltenere Indikationen. In den 90er Jahren hat sich der Indikationsbereich auch auf Patienten mit akuter respiratorischer Insuffizienz oder mit Schwierigkeiten bei der Entwöhnung vom Respirator ausgeweitet. Dabei liegen vor allem gute Daten für die Akutexazerbation der COPD vor [2-5].

Durch die Heimbeatmung kommt es bei einem Großteil der Patienten zu einer Verbesserung des Gasaustausches und der Symptome wie Dyspnoe, morgendliche Kopfschmerzen, Schläf- 


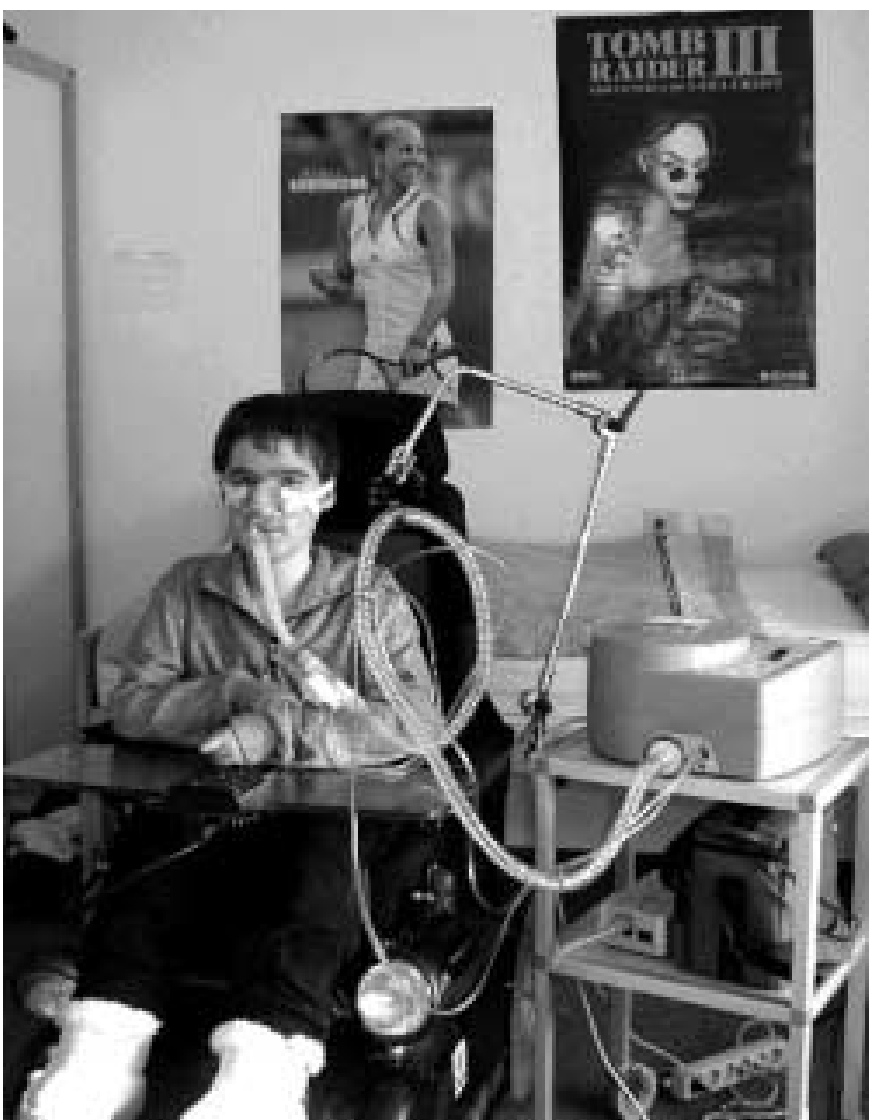

Abb. 1 Patient mit Muskeldystrophie Duchenne und nasaler Maskenbeatmung.

rigkeit, Schlappheit, Albträume oder Zeichen der chronischen Rechtsherzbelastung. Als mögliche Mechanismen werden hierfür drei verschiedene Zustände diskutiert:

- eine intermittierende Entlastung der übermüdeten Atemmuskulatur mit anschließender Funktionsverbesserung derselben,

- eine Verbesserung der pulmonalen Compliance mit Verringerung von Mikroatelektasen und daher der Atemarbeit und

- eine Re-Sensibilisierung des Atemzentrums für Kohlendioxid, verbunden mit einer renalen Elimination erhöhter Bikarbonatkonzentrationen.

Für Patienten mit thorakal-restriktiven und neuromuskulären Erkrankungen gilt nach dem aktuellen Wissenstand ein deutlicher Überlebensvorteil durch die Heimbeatmung als gesichert [6,7]. Allerdings liegen hierzu keine kontrollierten Studien vor, da sich diese aus ethischen Gründen verbieten. Für Patienten mit COPD wird ein Überlebensvorteil noch kontrovers diskutiert. Ein gesicherter Überlebensvorteil, wie er durch eine Sauerstofflangzeittherapie belegt ist, konnte bis dato für COPD-Patienten noch nicht nachgewiesen werden. Dabei scheint das Langzeitüberleben unter einer Heimbeatmung aber vergleichbar mit dem Langzeitüberleben unter einer Sauerstofflangzeittherapie zu sein.

\section{Lebensqualitätsforschung}

Die Lebensqualität eines Patienten hat in den letzten Jahren als neues Behandlungskriterium entscheidendes Gewicht bei der Beurteilung des medizinischen Therapieerfolges erhalten. Die Lebensverlängerung stellt somit nicht mehr das einzige Ziel therapeutischer Maßnahmen dar. Vielmehr wird der subjektiven Sicht des Patienten bezüglich seiner Befindlichkeit in verschiedenen Bereichen des Lebens eine zunehmend wichtigere Rolle beigemessen [8]. Diese neue Sichtweise hat seit den 80er und mehr noch in den 90er Jahren des letzten Jahrhunderts großen Einfluss auf die klinische Forschung erhalten, was durch die ständig wachsende Zahl wissenschaftlicher Publikationen zu diesem Thema zum Ausdruck kommt $[9,10]$.

Diese so genannte Lebensqualitätsforschung ist ein interdisziplinäres Feld, das Mediziner, Psychologen, Statistiker, Ökonomen und verschiedene Experten der Administration des Gesundheitswesens vereint [8]. Sie geht von einem weitgefassten biopsychosozialen Krankheitsmodell aus, bei dem auf dem Boden der subjektiven Einschätzung des Patienten innerpsychische Regulations- und Bewertungsprozesse evaluiert werden [8]. Da dieser Ansatz in erster Linie gesundheitsbezogene Aspekte fokussiert, wird Lebensqualität in dieser Begriffsdefinition abgegrenzt von der Lebensqualität aus materieller, ökonomischer, sozialer, politischer und religiöser Perspektive. Man spricht daher von der gesundheitsbezogenen Lebensqualität (health-related quality of life) [11].

\section{Die gesundheitsbezogene Lebensqualität (health-related quality of life)}

Der Begriff der gesundheitsbezogenen Lebensqualität ist in der Literatur allerdings nicht eindeutig definiert [12-14]. Es besteht aber international zunehmend Einigkeit darüber, dass die gesundheitsbezogene Lebensqualität als psychologisches Konstrukt zu verstehen ist, das in einem mehrdimensionalen Modell das subjektive Befinden eines Patienten auf vier minimal zu definierenden Grundsäulen charakterisiert, nämlich unter Berücksichtigung physischer, psychischer, sozialer und funktionaler Aspekte $[9,11]$. Diese subjektive Wahrnehmung des Patienten wird nicht nur vom Charakter der Grunderkrankung, sondern auch entscheidend vom Verlauf derselben wie auch von der Behandlung beeinflusst. So ist die Bewertung der Nebenwirkungen einer zytostatischen Chemotherapie abhängig davon, ob ein kuratives oder ein palliatives Therapieziel angestrebt wird $[15,16]$. Daneben kann Lebensqualität abhängig von einer optimistischen bzw. pessimistischen Grundeinstellung deutlich unterschiedlich bewertet werden [15]. Ebenso sind Vorerfahrungen, Erwartungen und persönliche Einstellungen individuell verschieden (perception of health) $[9,11]$.

Durch die zunehmende internationale Zusammenarbeit auf dem Gebiet der Lebensqualitätsforschung stellt sich zwangsläufig auch die Frage, ob interkulturell ein einheitliches Lebensqualitätskonstrukt besteht. Es könnte z.B. eine kulturelle Universalität dahingehend bestehen, dass es unabhängig von der Nationalität für den Patienten wichtig ist, keine Krankheitssymptome zu haben, psychisch ausgeglichen zu sein, soziale Integrität zu genießen und frei von funktionellen Einschränkungen zu sein. Un- 
klar bleibt jedoch dann die Frage der Gewichtung einzelner Dimensionen und Unterdimensionen des Konstruktes Lebensqualität im interkulturellen Vergleich [17].

Methoden zur Erfassung der gesundheitsbezogenen Lebensqualität

\section{Messinstrumente}

Bei der Erfassung der gesundheitsbezogenen Lebensqualität steht die subjektiv empfundene Befindlichkeit im Vordergrund. Diese Entwicklung trägt einer konsequenten Patientenorientierung Rechnung. Mit Erfassung der gesundheitsbezogenen Lebensqualität wird das Erleben des Patienten fokussiert. Dieser Aspekt der Patientenorientierung findet unter methodischen $\mathrm{Ge}$ sichtspunkten darin seinen Ausdruck, dass Fragebogen bei der Erfassung der gesundheitsbezogenen Lebensqualität dominieren. Alternativ kann ein standardisiertes Interview eingesetzt werden. Eine Fremdbeurteilung durch ärztliches Personal oder Angehörige kann die Eigenbeurteilung ergänzen, diese aber nicht ersetzten. Fragebogen eignen sich der guten Vergleichbarkeit wegen insbesondere für den Einsatz bei großen Patientenkollektiven. Dagegen können Einzelfallstudien die Lebensqualität dann zielsicherer beschreiben, wenn individuelle, komplexe und ggf. seltene Faktoren nachvollzogen werden sollen, die durch einen Fragebogen nicht erfasst werden können. Als weitere Methoden werden Therapieabbruchraten und Selbstbeobachtungen (Tagesprotokoll) diskutiert [8].

\section{Test-Gütekriterien von Fragebogen zur Messung der gesundheitsbezogenen Lebensqualität}

Ein Fragebogen zur Messung der gesundheitsbezogenen Lebensqualität muss definierten Anforderungen und Test-Gütekriterien genügen [18 - 20]. Neben der Objektivität des Testes, also der Unabhängigkeit der Untersuchungsergebnisse vom Untersucher, wird ein hoher Grad an Genauigkeit des Messinstrumentes, mit dem ein Merkmal gemessen wird, gefordert (Reliabilität). Die Reliabilität eines Messinstrumentes kann unterschiedlich berechnet werden. So ergibt sich die Test-Retest-Reliabilität aus den Korrelationen von Testergebnissen zu mindestens zwei verschiedenen Zeitpunkten; am häufigsten wird die interne Konsistenz (nach Cronbach's Alpha) angegeben, die den inneren Zusammenhang der einzelnen Fragen angibt. Wichtigstes Gütekriterium bildet die Validität. Diese gibt an, wie genau der Sachverhalt, den es zu messen gilt, tatsächlich gemessen werden kann. Die Validität umfasst unterschiedliche Aspekte [11,19]:

- Inhaltliche Validität (content validity): Der Test selbst stellt das optimale Kriterium für das Persönlichkeitsmerkmal oder die Verhaltensweise dar. Die inhaltliche Validität wird in der Regel durch Erarbeiten des Instrumentes zusammen mit Experten angestrebt („Experteninterview“) [21].

- Konstruktvalidität (construct validity): Hierbei werden Beschreibungsmerkmale erfasst, die nicht in eindeutiger Weise operational erfassbar sind, sondern theoretischen Charakter haben oder sich eher über ein psychologisches Konstrukt beschreiben lassen (z. B. Angst, Lebensqualität, Intelligenz). Der Test wird dahingehend überprüft, ob er ein solches „Konstrukt“ zu erfassen vermag. Dies kann durch eine Faktorenanalyse geschehen.
- Kriterienbezogene Validität (criterion validity): Die Testergebnisse werden mit einem Außenkriterium korreliert. Eine weitere sehr einfache Möglichkeit der Validierung ergibt sich in diesem Zusammenhang, wenn man durch ein Erhebungsinstrument unterschiedliche Gruppen (bekannte klinische Extremgruppen) präzise beschreiben kann.

\section{Krankheitsübergreifende und krankheitsspezifische Fragebogen}

Man kann zwischen krankheitsübergreifenden (krankheitsunspezifischen) Fragebogen und krankheitsspezifischen Fragebogen zur Messung der gesundheitsbezogenen Lebensqualität unterscheiden $[8,9]$. Erstere sind historisch älter und erlauben einen Vergleich zwischen verschiedenen spezifischen Gruppen. Einige dieser Fragebogen ermöglichen darüber hinaus den Vergleich mit Normkollektiven. Die Universalität des Messinstrumentes wird mit seiner fehlenden Spezifität erkauft. Das bedeutet, dass bei spezifischen Fragestellungen krankheitsübergreifende Fragebogen nur unzureichend die Lebensqualität erfassen können. Darüber hinaus verfügen sie über eine eingeschränkte Änderungssensibilität. So können Veränderungen der Lebensqualität durch eine medizinische Intervention nur unscharf abgebildet werden, wenn ausschließlich krankheitsübergreifende Fragebogen eingesetzt werden.

Im Gegensatz dazu stehen Instrumente, die erkrankungsspezifische Aspekte der gesundheitsbezogenen Lebensqualität erfassen und daher in der Regel therapiebedingte Veränderungen der Lebensqualität genauer erfassen. So können z.B. beim Asthma bronchiale Veränderungen der gesundheitsbezogenen Lebensqualität durch die Einleitung einer medikamentösen Therapie besser erfasst werden, wenn der Fragebogen spezifisch nach Symptomen, Einschränkungen und Behinderungen fragt, die eng mit der Erkrankung assoziiert sind [22,23]. Der Nachteil krankheitsspezifischer Messinstrumente liegt entsprechend in der fehlenden Universalität, denn je spezifischer ein Messinstrument ist, desto kleiner ist sein Einsatzbereich. Dennoch sind krankheitsspezifische Messinstrumente zentral, wenn es um die Beurteilung von speziellen Therapiestrategien geht, so dass in den letzten Jahren für eine Vielzahl von Erkrankungen spezifische Fragebogen zur Beurteilung der gesundheitsbezogenen Lebensqualität entwickelt wurden. Die Fragebogen, die bei Patienten mit Heimbeatmung im Rahmen von Studien eingesetzt wurden, sind in Tab. 1 aufgeführt.

\section{Krankheitsübergreifende Fragebogen, die bei Heimbeatmung eingesetzt worden sind}

Zu den ersten gut validierten und häufig eingesetzten Fragebogen gehören das Nottingham Health Profile (NHP) [24] mit 28 Fragen in 6 Subskalen sowie das Sickness Impact Profile (SIP) $[25,26]$. Letzteres ist mit 136 Fragen in 12 Bereichen der gesundheitsbezogenen Lebensqualität eines der umfangreichsten Instrumente überhaupt. Übersetzungen liegen in mehreren Sprachen vor. Die Werte entsprechen dem Prozentsatz der maximalen Einschränkung, hohe Werte reflektieren folglich eine starke Einschränkung. Das MOS 36-Item Short-Form Health Survey (SF-36) [27 - 30] enthält 36 Fragen in 8 Bereichen der subjektiven Gesundheit. Er ist gegenwärtig der wohl bekannteste und am meisten eingesetzte krankheitsübergreifende Fragebogen. Seine internationale Verfügbarkeit basiert auf der Arbeit der Interna- 
Tab. 1 Fragebogen, die bei Patienten mit Heimbeatmung eingesetzt wurden

\begin{tabular}{|c|c|c|c|c|}
\hline Fragebogen & Abkürzung & Literatur & Skalen & Zielgruppe \\
\hline $\begin{array}{l}\text { MOS 36-Item Short-Form } \\
\text { Health Survey }\end{array}$ & SF-36 & {$[27-35]$} & 8 & $\begin{array}{l}\text { krankheits- } \\
\text { übergreifend }\end{array}$ \\
\hline Sickness Impact Profile & SIP & {$[25,26]$} & 12 & $\begin{array}{l}\text { krankheits- } \\
\text { übergreifend }\end{array}$ \\
\hline $\begin{array}{l}\text { Nottingham Health } \\
\text { Profile }\end{array}$ & NHP & [24] & 6 & $\begin{array}{l}\text { krankheits- } \\
\text { übergreifend }\end{array}$ \\
\hline $\begin{array}{l}\text { Hospital Anxiety and } \\
\text { Depression Scale }\end{array}$ & $\mathrm{HAD}$ & [36-39] & 2 & $\begin{array}{l}\text { krankheits- } \\
\text { übergreifend }\end{array}$ \\
\hline $\begin{array}{l}\text { Mood Adjective Check } \\
\text { List }\end{array}$ & $\mathrm{MACL}$ & [46] & 3 & $\begin{array}{l}\text { krankheits- } \\
\text { übergreifend }\end{array}$ \\
\hline $\begin{array}{l}\text { Chronic Respiratory } \\
\text { Disease Questionnaire }\end{array}$ & CRQ & [40] & 4 & COPD \\
\hline $\begin{array}{l}\text { St. George's Respiratory } \\
\text { Questionnaire }\end{array}$ & SGRQ & {$[20,41]$} & 3 & COPD \\
\hline $\begin{array}{l}\text { Fragebogen zur chroni- } \\
\text { schen Heimbeatmung }\end{array}$ & $\mathrm{FCH}$ & [42] & 5 & Heimbeatmung \\
\hline Dresdner Fragebogen & & [62] & & $\begin{array}{l}\text { Heimbeatmung } \\
\text { im Kindesalter }\end{array}$ \\
\hline $\begin{array}{l}\text { Maugeri Foundation } \\
\text { Respiratory Failure } \\
\text { item set }\end{array}$ & MRF-28 & [43] & 3 & $\begin{array}{l}\text { chronische } \\
\text { respiratorische } \\
\text { Insuffizienz }\end{array}$ \\
\hline $\begin{array}{l}\text { Severe Respiratory } \\
\text { Insufficiency } \\
\text { Ouestionnaire }\end{array}$ & SRI & [44] & 7 & Heimbeatmung \\
\hline
\end{tabular}

tional Quality of Life Assessment Group (IQOLA) [17,31 - 35]. Danach wird ein Studienprotokoll erarbeitet, was die Übersetzung in andere Sprachen sowie die psychometrische Prüfung und Normierung festlegt. Die Hospital Anxiety and Depression Scale (HAD-Skala) wurde als psychologisches Screening-Instrument zur Erfassung von Angst und Depression bei Patienten internistischer Kliniken entwickelt, wobei auch eine deutsche Fassung vorliegt [36-39]. Dabei zeigt die Skala keine Interferenz mit somatischen Symptomen von Angst und Depression, so dass physische Einschränkungen wie z.B. Schlafstörungen die Ergebnisse nicht verfälschen.

\section{Krankheitsspezifische Fragebogen, die bei Heimbeatmung eingesetzt worden sind}

Für Patienten mit COPD wurden in der Vergangenheit mehrere spezifische Messinstrumente entwickelt. Zwei davon aus dem angloamerikanischen Sprachraum wurden bei Patienten mit COPD und Heimbeatmung eingesetzt: Der Chronic Respiratory Disease Questionnaire (CRQ) mit 4 Ebenen (Dyspnoe, Müdigkeit, emotionale Funktionen, Lebensbewältigung) [40] und der St. George's Respiratory Questionnaire (SGRQ) mit 3 Bereichen (Symptome, Aktivität, psychische Beeinträchtigungen) [41]. Eine autorisierte deutsche Übersetzung des SGRQ liegt seit 1999 vor [20]. Allerdings wurden diese Fragebogen an COPD-Patienten mit sehr unterschiedlichen Schweregraden ihrer Erkrankung entwickelt, so dass zu hinterfragen bleibt, ob diese Fragebogen für Patienten mit COPD und schwerer respiratorischer Insuffizienz geeignet sind.

Aus diesem Grund wurden in letzten Jahren Fragebogen speziell für Patienten mit schwerer respiratorischer Insuffizienz entwi- ckelt. Der erste war der Fragebogen zur chronischen Heimbeatmung (FCH) mit 57 Fragen [42]. Neben psychischen, sozialen und allgemein funktionalen Aspekten der Lebensqualität konzentriert sich der Fragebogen auf funktionale Aspekte unter Beatmung und Aspekte, die Nebenwirkungen der Beatmung enthalten. Ein eigener Teil des Fragebogens erfasst retrospektiv Veränderungen der Lebensqualität. Die Fragen wurden mit dem Ziel einer hohen inhaltlichen Validität nach Ergebnissen einer Patientenbefragung aufgestellt. In einer ersten Prüfung konnte in Teilbereichen eine konvergente Validität nachgewiesen werden.

Außerdem wurde das Maugeri Foundation Respiratory item set (MRF-28) für Patienten mit chronischer respiratorischer Insuffizienz entwickelt [43]. Dieser Fragebogen wurde im Rahmen einer Studie unter Einschluss von Patienten mit Heimbeatmung aber auch mit Sauerstofflangzeittherapie entworfen, wobei neben neuen Fragen auch solche aus älteren gut validierten Fragebogen (u. a. SGRQ, CRQ, SIP) mit aufgenommen wurden. Der Fragebogen enthält 28 Fragen (items) in drei Bereichen: Alltagsaktivität, kognitive Funktionsfähigkeit und Invalidität. Der MRF-28 verfügt über eine hohe inhaltliche Validität. Nachteilig sind jedoch eine geringe Varianzaufklärung bei der Konstruktvalidität sowie die Tatsache, dass der Fragebogen vor allem an COPD-Patienten validiert wurde. Der Einsatz bei Patienten mit restriktiven Erkrankungen oder neuromuskulären Erkrankungen ist daher nicht unproblematisch. Eine validierte deutsche Übersetzung steht aktuell nicht zur Verfügung.

\section{Der Fragebogen zur Befindlichkeit bei schwerer respiratorischer Insuffizienz (SRI)}

Auf dem Boden der Erfahrungen mit dem FCH wurde in der eigenen Arbeitsgruppe der Severe Respiratory Insufficiency (SRI) Questionnaire mit 49 Fragen in 7 Subskalen (Atembeschwerden, körperliche Rollenfunktion, Begleitsymptome/Schlaf, soziale Beziehungen, krankheitsbezogene Ängste, psychische Befindlichkeit und soziale Rollenfunktion) in deutscher Sprache entwickelt (Abb. 2) [44]. Im Rahmen einer multizentrischen Validierungsstudie unter Einschluss von 226 Patienten mit chronischer respiratorischer Insuffizienz unterschiedlicher Genese und Heimbeatmungstherapie wurden für den SRI hohe psychometrische Kennwerte nachgewiesen: Die interne Konsistenz war mit einem Cronbach's Alpha von $>0,7$ in allen Skalen und $>0,8$ in vier der sieben Subskalen ein Maß für die hohe Reliabilität. Eine inhaltli-

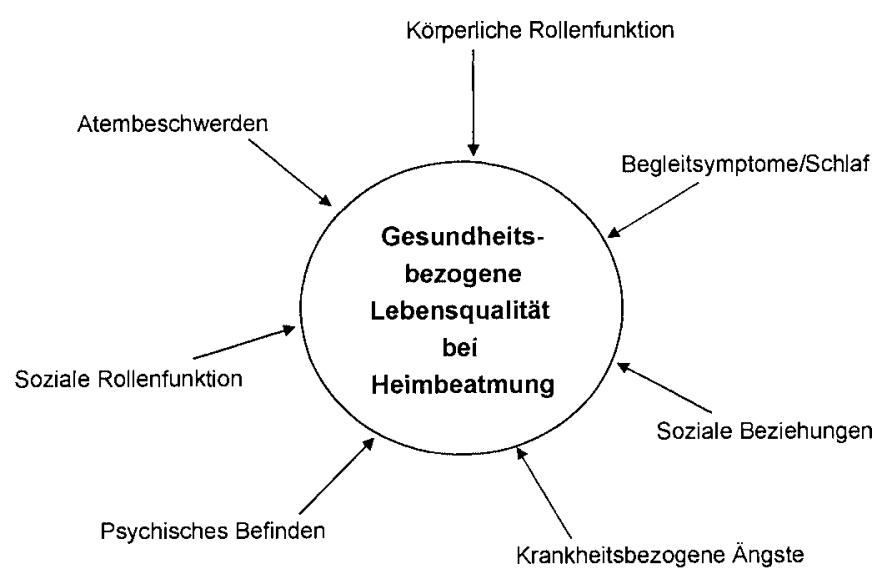

Abb. 2 Messmodell des Fragebogens zur Befindlichkeit bei Schwerer Respiratorischer Insuffizienz (SRI) 
che Validität war dadurch gegeben, dass die Fragen durch ein Expertengremium entwickelt wurden (Pneumologen, Psychologen). Darüber hinaus konnte durch Korrelationsanalysen mit dem SF-36 eine gute Übereinstimmung sowie über eine Faktorenanalyse eine Konstruktvalidität nachgewiesen werden, wobei eine Summenskala aus den 7 Subskalen gebildet wurde [44].

Alle Fragen werden auf einer 5-Punkte-Likert-Skala beantwortet. Um Ja-Sage-Tendenzen zu vermeiden, entsprechen je nach Frage hohe oder tiefe Werte einem günstigen Resultat. In der Auswertung werden die Fragen einheitlich umkodiert. Für jede Subskala und für die Summenskala wird ein Score zwischen 0 und 100 errechnet, wobei hohe Werte eine höhere Lebensqualität im Sinne des Konstruktes der Skala und tiefe Werte entsprechend eine niedrigere Lebensqualität widerspiegeln.

\section{Praktischer Einsatz von Fragebogen zur Messung der gesundheitsbezogenen Lebensqualität bei Heimbeatmung}

Grundsätzlich wird zur Messung der gesundheitsbezogenen Lebensqualität eines definierten Patientenkollektivs der Einsatz sowohl eines spezifischen als auch eines unspezifischen Messinstrumentes empfohlen, um die Vorteile der Universalität und Vergleichbarkeit einerseits sowie der Spezifität und Änderungssensibilität andererseits zu vereinen. Das Beantworten von Fragebogen ist jedoch bei Patienten mit Heimbeatmung begrenzt. Zum einen ist ein Teil der Patienten mit einer generalisierten Muskelschwäche nicht in der Lage, Kreuze auf dem Antwortbogen zu markieren. Zum anderen kann das Ankreuzen zu vieler Fragen für Patienten mit fortgeschrittenem Leiden nicht zumutbar sein. Dabei wird die Anstrengung und notwendige Konzentration dafür nicht selten unterschätzt. Das betrifft vor allem Fragebogen mit vielen Fragen (z. B. SIP [26] mit 136 Fragen) und insbesondere Patienten vor Einleitung einer Heimbeatmung. Eine Reduzierung der Fragen oder die Durchführung eines Interviews können dann sinnvoll sein. In Einzelfällen können auch Blindheit oder mentale Einschränkungen das Ausfüllen von Fragebogen unmöglich machen. Dann können Angehörige oder der Untersucher selbst bei der Beantwortung der Fragen helfen, was allerdings die Objektivität beeinträchtigen kann.

Als krankheitsübergreifender Fragebogen eignet sich für den deutschen Sprachraum gegenwärtig am besten der SF-36 [27-30], da er kürzer als die meisten anderen Fragebogen ist, hervorragend validiert ist und darüber hinaus einen internationalen Vergleich mit Normkollektiven und anderen PatientenGruppen erlaubt. Die HAD-Skala [36-39] ist kurz und einfach auszufüllen, sie kann daher zusätzlich eingesetzt werden, wenn es um die spezielle Erfassung von Ängsten und Depressionen geht. Bei COPD-Patienten kann der SGRQ [41] als spezifisches Instrument eingesetzt werden. Als krankheitsspezifischer Fragebogen für die schwere respiratorische Insuffizienz eignet sich am besten der SRI [44], insbesondere, wenn Patienten mit verschiedenen Grunderkrankungen vergleichend betrachtet werden.
Gesund lheitsbezogene Lebensqualität bei Heimbeatmung aktueller Stand

\section{Gesundheitsbezogene Lebensqualität bei Heimbeatmung im Vergleich zu Normalkollektiven und zu anderen Patientengruppen}

Die erste Arbeit, die sich intensiv mit der Lebensqualität bei Heimbeatmungs-Patienten beschäftigt hat, war die schwedische Studie von Pehrsson u. Mitarb. von 1994. In dieser Studie wurden 39 Patienten mit einer chronischen ventilatorischen Insuffizienz auf dem Boden einer restriktiven Ventilationsstörung untersucht. Neben dem SIP [26], der HAD-Skala [36], einem allgemeinen Teil des EORTC Quality of Life Questionnaire (QLQ-C30) [45] und der Mood Adjektive Check List (MACL) [46] wurde ein eigener Fragebogen eingesetzt, der mit 57 Fragen in 3 Ebenen (Sozialleben, Probleme mit dem Beatmungsgerät, Schlafqualität) speziell für die Studie entwickelt wurde. Zusammenfassend konnte aus der Vielzahl der Ergebnisse dieser Studie eine hohe gesundheitsbezogene Lebensqualität insbesondere für den psychosozialen Bereich abgeleitet werden. Zwar zeigten sich im SIP vermehrte Einschränkungen im Vergleich zu einer gesunden Referenzgruppe, verglichen mit anderen Patientengruppen, z. B. Patienten mit chronischen Rückenschmerzen oder rheumatoider Arthritis [47], waren diese Einschränkungen jedoch geringer ausgeprägt. Patienten mit neuromuskulären Erkrankungen waren physisch und psychosozial von allen Beatmungspatienten noch am stärksten limitiert. Insgesamt lief die Beatmung mit nur wenigen Problemen, die meisten Patienten waren mit der Beatmungstherapie deutlich zufrieden. Die Schlafqualität wurde insgesamt als gut bezeichnet. Auch die Auswertung der HAD-Skala konnte keine erhöhte Prävalenz von Angst und Depression aufzeigen.

Die Interpretation dieser Ergebnisse muss jedoch vor dem Hintergrund geschehen, dass die guten Ergebnisse auch eine Selektion des Patientenkollektivs reflektieren. Zum einen betrug die Dauer der Heimbeatmung im Mittel 50 Monate, so dass in erster Linie Patienten eingeschlossen wurden, die dauerhaft motiviert waren. Zum anderen war die tägliche Beatmungszeit mit durchschnittlich 7,7 Stunden trotz der langen Therapiedauer eher niedrig. Der tendenziell gute Allgemeinzustand der Patienten kommt auch dadurch zum Ausdruck, dass alle Befragten in der Lage waren, die insgesamt ca. 250 Fragen zu beantworten. Für viele andere Patienten könnte dies eine nicht überwindbare Mühe bedeuten. Schließlich wurden keine Patienten mit COPD in die Studie eingeschlossen.

In der großen Studie von Simonds u. Mitarb. wurden 180 Patienten untersucht, die auf eine Heimbeatmung eingeleitet wurden [7]. Bei den 105 Patienten, die in einer Subgruppe von insgesamt 118 Patienten den SF-36 beantwortet haben, konnte gezeigt werden, dass die gesundheitsbezogene Lebensqualität bei Heimbeatmung zwar deutlich eingeschränkt ist im Vergleich zu einem Normalkollektiv [7]. Diese Einschränkungen waren jedoch nicht wesentlich deutlicher als bei Patienten mit anderen chronischen Erkrankungen.

In der Validierungsstudie des SRI wurde außerdem der SF-36 eingesetzt, um eine Aussage zur Ähnlichkeit der Verfahren zur Messung der gesundheitsbezogenen Lebensqualität zu machen 


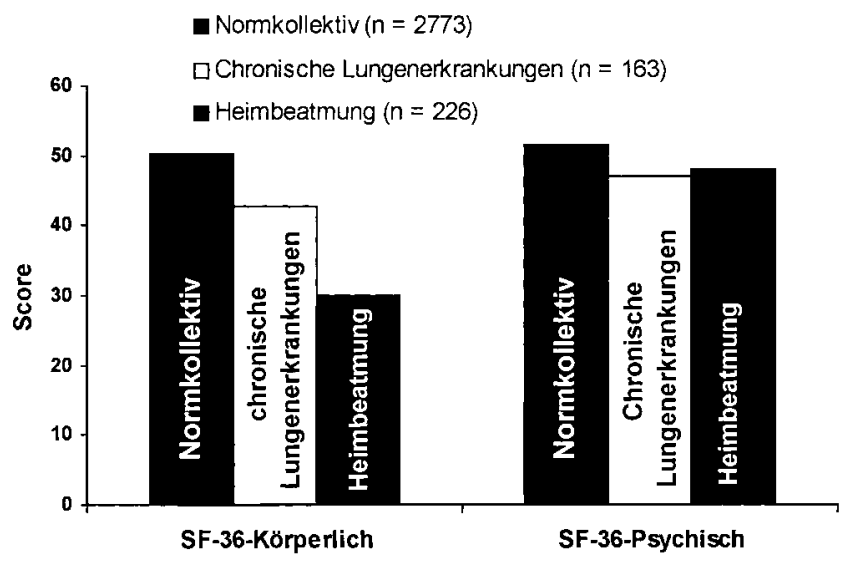

Abb. 3 Scores für die beiden Summenskalen (körperliche Gesundheit und seelische Gesundheit) des SF-36 in einer Querschnittsstudie bei 226 Patienten mit Heimbeatmung im Vergleich mit einem historischen Normalkollektiv und einem historischen Kollektiv mit chronischen Lungenerkrankungen, die nicht abhängig von einem Respirator sind (100 = maximal hohe Lebensqualität; 0 = niedrigste Lebensqualität).

(konvergente Validität, s. o.) [44]. Dabei wurde aber in den Summenskalen des SF-36 auch deutlich, dass die körperliche Gesundheit von Patienten mit Heimbeatmung im Vergleich zu einem Normalkollektiv und einem Patientenkollektiv mit chronischen Lungenerkrankungen [30] deutlich eingeschränkt ist, was aufgrund der oftmals deutlichen und offensichtlichen körperlichen Behinderungen der Patienten zu erwarten ist (Abb. 3). Allerdings zeigt sich im Vergleich dieser Untersuchungsgruppen für Patienten mit Heimbeatmung keine richtungsweisende Einschränkung in der psychischen Summenskala. Daraus ergibt sich, dass sogar schwere körperliche Beeinträchtigungen, wie sie bei Patienten mit Heimbeatmung vorliegen, nicht notwendigerweise zu psychischen Beeinträchtigungen führen müssen. Diese Ergebnisse entsprechen denen in der Studie von Pehrsson u. Mitarb. [48].

Zusammenfassend bestehen deutliche Einschränkungen der gesundheitsbezogenen Lebensqualität bei Patienten mit Heimbeatmung im Vergleich zu Gesunden. Diese Einschränkungen sind jedoch nicht notwendigerweise größer als bei anderen Patienten, die nicht von einem Beatmungsgerät abhängig sind. Darüber hinaus betreffen die Einschränkungen der Lebensqualität in erster Linie die körperliche Ebene. Dies führt nicht zwangsläufig, wie häufig geglaubt, auch zu psychischen Veränderungen, da das psychische Befinden bei vielen Patienten mit Heimbeatmung trotz schwerer körperlicher Beeinträchtigungen normal sein kann.

\section{Unterschiede in der gesundheitsbezogenen Lebensqualität bei verschiedenen Patienten-Gruppen mit Heimbeatmung}

In der aktuellen Studie von Dellborg u. Mitarb. zeigen sich Unterschiede in der gesundheitsbezogenen Lebensqualität bei einem gemischten Patienten-Kollektiv mit chronischem Hypoventilations-Syndrom auf dem Boden thorakal-restriktiver Erkrankungen, die noch nicht auf eine Heimbeatmung eingeleitet worden sind [49]. Dabei hatten Patienten mit posttuberkulösem Syndrom eine schlechtere gesundheitsbezogene Lebensqualität als Patienten mit Kyphoskoliose oder Post-Polio-Syndrom, was insbesondere auf der psychosozialen Ebene deutlich war. Allerdings ist die Studie durch ihre eher geringe Fallzahl $(\mathrm{n}=44)$, durch die heterogene Anzahl von Patienten in den einzelnen Krankheitsgruppen sowie durch die Tatsache limitiert, dass die Patientengruppen nicht für Alter und Geschlecht kontrolliert werden konnten, zumal fortgeschrittenes Alter insbesondere ab 70 Jahren als negativer Vorhersagewert vor allen Dingen für die körperliche Gesundheit angesehen werden darf [50]. Bemerkenswert bleibt jedoch, dass sich diese Ergebnisse bei Patienten mit chronischer ventilatorischer Insuffizienz ohne Heimbeatmung decken mit früheren Ergebnissen bei Patienten mit chronischer ventilatorischer Insuffizienz und langfristiger Heimbeatmung in Bezug auf krankheitsbedingte Unterschiede in der gesundheitsbezogenen Lebensqualität [48]. Einschränkungen in der gesundheitsbezogenen Lebensqualität bei Patienten mit posttuberkulösem Syndrom im Vergleich zu anderen Patienten mit thorakalrestriktiven Erkrankungen scheinen somit unabhängig von der Heimbeatmung krankheitsspezifisch zu sein.

In der großen Studie von Simonds u. Mitarb. [7] konnte anhand des SF-36 für Patienten mit COPD insgesamt eine schlechtere Lebensqualität nachgewiesen werden im Vergleich zu Patienten mit thorakal-restriktiven Erkrankungen. Auf der funktionalen Ebene waren neuromuskulär Erkrankte am stärksten limitiert, was darin zu sehen ist, dass diese Patienten neben den funktionellen Einschränkungen durch die Atemstörung natürlich auch durch die allgemeine Muskellähmung funktionell limitiert sind. Allerdings sind in dieser Studie die einzelnen Subskalen des SF-36 in den verschiedenen Patienten-Gruppen nicht weiter quantifiziert, so dass eine weitere Analyse in dieser Studie nicht möglich ist.

In einer anderen Studie wurden insgesamt 67 Patienten mit Heimbeatmung zu ihrer gesundheitsbezogenen Lebensqualität befragt [42]. Dabei wurde in retrospektiver Betrachtung ein Gruppenvergleich zwischen verschiedenen Patientengruppen angestrebt. Die tägliche Beatmungsdauer betrug im Mittel bei COPD-Patienten 9,5 Stunden, bei Skoliose-Patienten 8,7 Stunden und bei neuromuskulär Erkrankten 10,5 Stunden. Die mittlere Beatmungsdauer in Monaten lag bei COPD-Patienten bei 27, bei Skoliose-Patienten bei 28 und bei Patienten mit neuromuskulären Erkrankungen bei 50. Auch in dieser Studie kamen mehrere Instrumente zum Einsatz: Der FCH [42], die HAD-Skala [36 - 39] sowie eine modifizierte Version des Asthma Quality of Life Questionnaire (AQLQ) [22,23]. Die Ergebnisse dieser Untersuchung lassen deutliche Unterschiede bei den verschiedenen Grunderkrankungen erkennen.

Während COPD-Patienten tendenziell keine Veränderung ihrer gesundheitsbezogenen Lebensqualität unter Heimbeatmung im Vergleich zum Zeitpunkt vor Beatmungsbeginn aufweisen, verbessert sich diese von Skoliose-Patienten und neuromuskulär Erkrankten deutlich. Auf physischer Ebene kommt es neben einem Rückgang der Atemnot zu einer verbesserten Schlafqualität. Nebenwirkungen der Beatmung sind beim Langzeitgebrauch wenig einschränkend. Psychisch zeigen insbesondere COPD-Patienten stärkere Defizite. Im Vergleich zu der Arbeit von Pehrsson u. Mitarb. [48] dokumentierten sich insgesamt deutlich höhere Auffälligkeiten für Angst und Depression, was, wie oben beschrieben, am ehesten in der Auswahl des Patientenkollektivs begründet ist. Minderwertigkeit, fehlender Kontakt zu engen Familienmit- 


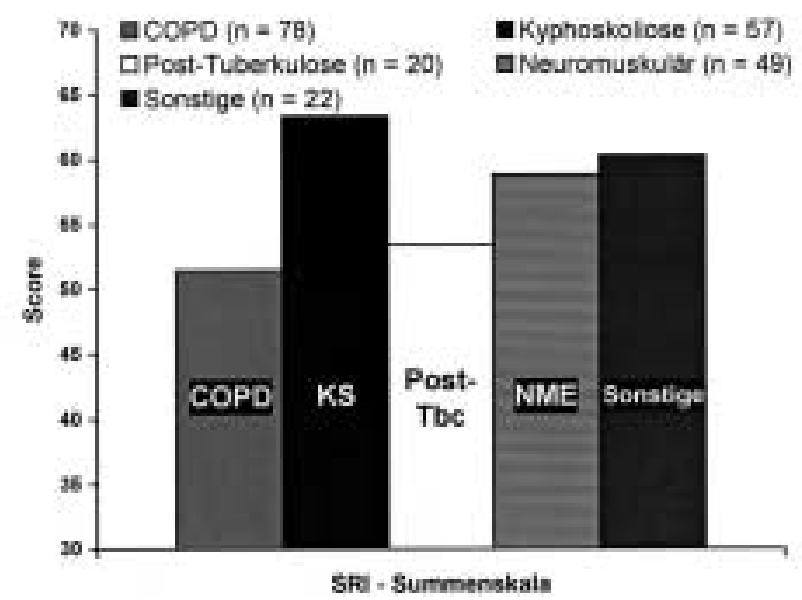

Abb. 4 Krankheitsgruppenvergleich mit dem Fragebogen zur Befindlichkeit bei Schwerer Respiratorischer Insuffizienz (SRI) in einer Querschnittsstudie bei 226 Patienten mit Heimbeatmung: Summenscore für die gesundheitsbezogene Lebensqualität (100 = maximal hohe Lebensqualität; $0=$ niedrigste Lebensqualität): $\mathrm{COPD}=$ Chronic obstructive pulmonary disease; $\mathrm{KS}=$ Kyphoskoliose; Post-Tbc $=$ Posttuberkulöses Syndrom; NME = Neuromuskuläre Erkrankungen; Sonstige $=$ Sonstige Erkrankungen .

gliedern oder andere Faktoren, die das soziale Befinden einschränken könnten, sind in allen Patientengruppen selten. Eine zusätzliche Angehörigenbefragung hat jedoch ergeben, dass nicht selten die Partner, die mit dem Patienten ein Schlafzimmer teilen, eine Störung durch das Geräusch des Beatmungsgerätes empfinden. In funktioneller Hinsicht sind insbesondere Patienten mit COPD und solche mit neuromuskulären Erkrankungen limitiert. Das betrifft vor allen Dingen Aktivitäten des Alltags wie Hausarbeit, Essen, sich Waschen oder Ankleiden.

In der Validierungsstudie des SRI wurden wie auch in den Untersuchungen mit dem FCH Unterschiede der Lebensqualität zwischen den verschiedenen Patientengruppen deutlich [44]. Dabei hatten Patienten mit Kyphoskoliose die insgesamt beste gesundheitsbezogene Lebensqualität im Summenscore (Abb.4). Am deutlichsten waren die Unterschiede im Bereich der krankheitsbezogenen Ängste, wobei Patienten mit COPD und posttuberkulösem Syndrom sehr viel deutlicher Ängste hatten als die anderen Patientengruppen. Dies deckt sich gut mit den Ergebnissen, die mittels HAD-Skala erarbeitet wurden, wo COPD-Patienten deutlich häufiger Ängste und Depressionen hatten als Patienten mit Kyphoskoliose oder neuromuskulären Erkrankungen [42]. Ähnlich war das Verhältnis bei den Atembeschwerden und bei der sozialen Rollenfunktion. Bei der körperlichen Rollenfunktion waren erwartungsgemäß Patienten mit neuromuskulären Erkrankungen am stärksten limitiert, jedoch weniger durch die Atembeschwerden als durch die Grunderkrankung selbst, da diese Patienten von allen Patientengruppen über die wenigsten Atembeschwerden berichtet hatten. In dieser Arbeit wie auch in anderen Untersuchungen hatte die Grunderkrankung somit den stärksten Einfluss auf die gesundheitsbezogene Lebensqualität, wobei abgesehen vom Alter [50] objektive Parameter wie Daten der Lungenfunktion und andere die gesundheitsbezogene Lebensqualität nur wenig voraussagen können. Am stärksten scheinen noch die erhöhten Bikarbonat-Werte mit Einschränkungen der gesundheitsbezogenen Lebensqualität zu korrelie- ren. Dies betrifft sowohl Patienten mit chronischer ventilatorischer Insuffizienz, die auf eine Heimbeatmung eingeleitet worden sind [48], als auch solche, die noch nicht beatmet werden [49].

Patienten mit Tracheotomie sind in der Regel stärker beeinträchtigt als solche, die über einen nichtinvasiven Beatmungszugang ventiliert werden. Dies betrifft insbesondere das Sprechen, das Essen, die Abhängigkeit von pflegenden Personen, den Komfort, Probleme, die sich aus dem künstlichen Beatmungszugang ergeben können sowie die Tatsache, dass auch im Falle der Beatmungspause häufig nicht über die natürlichen Atemwege geatmet werden kann [51]. Gelegentlich kann der sichere Beatmungszugang jedoch auch mehr Sicherheit und Vertrauen für den Patienten bedeuten.

Zusammenfassend ergibt sich, dass die gesundheitsbezogene Lebensqualität bei Patienten mit COPD deutlicher eingeschränkt ist als bei den meisten Patienten mit thorakal-restriktiven oder neuromuskulären Erkrankungen. Ähnlich scheint dies bei Patienten mit posttuberkulösem Syndrom der Fall zu sein. Das betrifft neben der physischen Funktion insbesondere auch psychische Aspekte wie Ängste und Depressionen. Dies liegt zumindest bei COPD-Patienten z.T. darin begründet, dass diese nicht nur durch ihre ventilatorische Insuffizienz, sondern auch in erheblichem Maße durch ihre pulmonale Insuffizienz beeinträchtigt sind. Dabei können ein Bronchospasmus, chronisch-produktiver Husten, die Hypoxie und die Folgen eines cor pulmonale die gesundheitsbezogene Lebensqualität zusätzlich zum Hypoventilations-Syndrom stark beeinträchtigen. Darüber hinaus kommt es bei COPDPatienten häufiger zu Infektexazerbationen, von denen gezeigt wurde, dass sie in der Folge die gesundheitsbezogene Lebensqualität erheblich beeinträchtigen können [52 - 54].

\section{Einfluss der Heimbeatmung auf die gesundheitsbezogene Lebensqualität in Verlaufsbeobachtungen}

Ein Vergleich zwischen historischen Patientenkollektiven in der Studie von Dellborg u. Mitarb. deutet an, dass sich die Lebensqualität bei Patienten mit verschiedenen thorakal-restriktiven Erkrankungen nach Einleitung der Heimbeatmung bessern kann [49]. Ein Teil der Einschränkungen der gesundheitsbezogenen Lebensqualität vor der Heimbeatmung kann somit auf die chronische ventilatorische Insuffizienz und nicht primär auf die Grunderkrankung selbst zurückgeführt werden. Diese Interpretation muss jedoch zurückhaltend erfolgen, da die Patienten ohne Heimbeatmung in der aktuellen Studie mit einem historischen Kollektiv von Patienten unter langfristiger Heimbeatmung verglichen werden.

Wie oben gezeigt, kommt es in der retrospektiven Betrachtung zu einem Gewinn an Lebensqualität bei Patienten mit thorakalrestriktiven und neuromuskulären Erkrankungen, nicht jedoch bei Patienten mit COPD [42]. Die retrospektive Betrachtung muss hier nicht unbedingt von Nachteil sein, da die aus der Rückschau subjektiv wahrgenommene Veränderung der Lebensqualität seit Beginn der Heimbeatmung für den Patienten ebenso wichtig sein kann wie die objektive Veränderung der Lebensqualität, die sich aus einer prospektiven Analyse ergibt. 
Dennoch wurden in der Vergangenheit Bemühungen unternommen, den Einfluss der Heimbeatmung prospektiv zu untersuchen. Hier wurden insbesondere COPD-Patienten, die auf eine Heimbeatmungstherapie eingeleitet wurden, mit unterschiedlichen Messinstrumenten prospektiv untersucht [55-58]. In zwei Arbeiten konnten keine wesentlichen Verbesserungen durch die Heimbeatmung dokumentiert werden $[55,58]$. In den anderen beiden Arbeiten zeigte sich eine signifikante Verbesserung der gesundheitsbezogenen Lebensqualität, wobei in beiden Studien im Gegensatz zu den ersten beiden u.a. der SGRQ eingesetzt wurde $[56,57]$. Fragebogen, die spezifisch die Probleme der Patienten mit Heimbeatmung erfragen, wurde jedoch in keiner dieser Studien eingesetzt, so dass der Effekt der Heimbeatmung auf die gesundheitsbezogene Lebensqualität weiterhin nicht sicher beurteilt werden kann.

In einer anderen Arbeit konnte bei 16 Patienten mit amyotropher Lateralsklerose unter Einsatz des SF-36 gezeigt werden, dass sich die gesundheitsbezogene Lebensqualität nach Einleitung einer Heimbeatmung trotz Progredienz der Erkrankung nicht verschlechtert und dass in der Subskala „Vitalität“ sogar ein Gewinn an Lebensqualität erzielt werden konnte [59]. Obwohl auch bei Patienten mit COPD, die mit Salmeterol behandelt wurden, und bei Patienten mit obstruktivem Schlafapnoe-Syndrom, die auf eine CPAP-Therapie eingeleitet wurden, Veränderungen der gesundheitsbezogenen Lebensqualität mittels SF-36 erfasst werden konnten [60,61], kann von diesem Fragebogen postuliert werden, dass er Veränderungen der gesundheitsbezogenen Lebensqualität weniger gut erfasst als ein krankheitsspezifischer Fragebogen, da er wesentliche Merkmale der Grunderkrankung nicht erfasst (s.o.). Die prospektive Untersuchung zur gesundheitsbezogenen Lebensqualität bei amyotropher Lateralsklerose [59] muss vor diesem Hintergrund kritisch betrachtet werden.

Zusammenfassend zeigen sich mehrere Studien, die den Einfluss der Heimbeatmung auf die gesundheitsbezogene Lebensqualität untersucht haben. In keiner Studie konnte bis dato eine Verschlechterung dokumentiert werden, was aufgrund der Progredienz vieler dieser Erkrankungen positiv zu bewerten ist. Allerdings bleibt nach wie vor ungeklärt, inwieweit sich die gesundheitsbezogene Lebensqualität unter der Heimbeatmung wirklich verbessern kann, da die prospektiven Studien mit hohen Patienten-Fallzahlen und Gebrauch moderner, spezifischer Messinstrumente noch ausstehend sind.

\section{Gesundheitsbezogene Lebensqualität bei Heimbeatmung im Kindesalter}

Die oben dargestellten Ergebnisse lassen aber nur Rückschlüsse auf die gesundheitsbezogene Lebensqualität bei Patienten mit Heimbeatmung im Erwachsenenalter zu, da Kinder und Jugendliche nicht in die Analysen miteinbezogen wurden. Daher wurde in einer Deutschland-weiten Multizenter-Studie die gesundheitsbezogene Lebensqualität bei Heimbeatmung im Kindesund Jugendalter untersucht [62], wobei eigens für die Studie ein spezifischer Fragebogen entwickelt wurde. Zusammenfassend ergab sich danach eine hohe Patientenzufriedenheit, wobei interessanterweise eine zusätzlich durchgeführte Eltern-/Angehörigenbefragung diskrepante Ergebnisse bezüglich der gesundheitsbezogenen Lebensqualität erarbeiten konnte und zwar in dem Sinne, dass die Patienten ihre Lebensqualität höher ein- schätzten als die Eltern. Dies unterstreicht, dass eine Angehörigenbefragung die Patientenbefragung niemals ersetzen kann.

\section{Zukünftige Studien zur Erfassung der Lebensqualität bei Patienten mit Heimbeatmung}

Wie oben aufgeführt, hat die Frage nach der Lebensqualität bei Patienten mit Heimbeatmung in zunehmendem Maße die wissenschaftliche Auseinandersetzung beschäftigt. Konklusive Aussagen zu dieser Frage können jedoch aktuell noch nicht gemacht werden, da in vielen Studien nicht die geeigneten Instrumente zur Erfassung der gesundheitsbezogenen Lebensqualität zur Verfügung standen. Nach gegenwärtigem Wissensstand sollten in zukünftigen Studien krankheitsübergreifende Fragebogen zum Vergleich mit anderen Patientengruppen mit krankheitsspezifischen Fragebogen zur Erfassung krankheitsspezifischer Aspekte der Grunderkrankung kombiniert werden. In diesem Sinne sollte auch der Wert der Heimbeatmung für die gesundheitsbezogene Lebensqualität prospektiv untersucht werden. Solche zukünftigen Forschungsvorhaben sollten zum Erreichen größerer Patienten-Fallzahlen multizentrisch sein, damit die Aussagekraft solcher Studien für zukünftige Therapieentscheidungen gegeben ist.

Aus diesem Grund wird aktuell eine Multizenter-Studie in Deutschland geplant, bei der der Einfluss der Heimbeatmung auf die gesundheitsbezogene Lebensqualität von Patienten mit schwerer respiratorischer Insuffizienz prospektiv mit dem SF-36 [27 - 30] und dem SRI [44] untersucht werden soll. Parallel dazu ist das internationale Projekt QuESS (The Quality of Life Evaluation and Survival Study) entstanden, das neben Überlebensraten auch die gesundheitsbezogene Lebensqualität u. a. unter Einsatz des MRF-28 [43] bei Patienten mit chronischer respiratorischer Insuffizienz prospektiv untersucht [63].

Die wissenschaftliche Beurteilung der gesundheitsbezogenen Lebensqualität im Verlauf einer Heimbeatmungstherapie ist insbesondere auch bei COPD-Patienten wichtig, da es in der Zukunft schwierig sein wird, die Indikation für eine aufwendige Therapie aufrechtzuerhalten, von der bis jetzt ein Vorteil weder für das Langzeitüberleben noch für die gesundheitsbezogene Lebensqualität gesichert ist.

\section{Literatul}

${ }^{1}$ Anonymous. Clinical indications for noninvasive positive pressure ventilation in chronic respiratory failure due to restrictive lung disease, COPD, and nocturnal hypoventilation - a consensus conference report. Chest 1999; 116: $521-534$

2 Mehta S, Hill NS. Noninvasive ventilation. Am J Respir Crit Care Med 2001; $163: 540-577$

${ }^{3}$ Brochard L, Mancebo J, Wysocki M et al. Noninvasive ventilation for acute exacerbations of chronic obstructive pulmonary disease. $\mathrm{N}$ Engl J Med 1995; 333: 817-822

${ }^{4}$ Nava S, Ambrosino N, Clini E et al. Noninvasive mechanical ventilation in the weaning of patients with respiratory failure due to chronic obstructive pulmonary disease. A randomized, controlled trial. Ann Intern Med 1998; 128: $721-728$

5 Plant PK, Owen JL, Elliott MW. Early use of non-invasive ventilation for acute exacerbations of chronic obstructive pulmonary disease on general respiratory wards: a multicentre randomised controlled trial. Lancet 2000; 355: 1931 - 1935 
${ }^{6}$ Leger P, Bedicam JM, Cornette A et al. Nasal intermittent positive pressure ventilation. Long-term follow-up in patients with severe chronic respiratory insufficiency. Chest 1994; 105: 100-105

${ }^{7}$ Simonds AK, Elliott MW. Outcome of domiciliary nasal intermittent positive pressure ventilation in restrictive and obstructive disorders. Thorax 1995; 50: 604-609

8 Petermann F, Bergmann KS. Lebensqualität und Asthma - eine Einführung. In: Petermann F, Bergmann KS. (Hrsg). Lebensqualität und Asthma. Berlin, München: Quintessenz, 1994: 9-16

9 Testa MA, Simonson DC. Assesment of quality-of-life outcomes. N Engl J Med 1996; 334: 835-840

10 Warschburger P. Lebensqualität und Compliance. In: Petermann F. (Hrsg). Compliance und Selbstmanagement. Göttingen: Hogrefe, 1998: $103-138$

${ }^{11}$ Bullinger M. Lebensqualität: Grundlagen und Anwendungen. In: Petermann F, Bergmann KC. (Hrsg). Lebensqualität und Asthma. Berlin, München: Quintessenz, 1994: 17-28

${ }^{12}$ Felce D, Perry J. Quality of life: its definition and measurement. Research in Developmental Disabilities 1995; 16: 51 - 74

13 Hoffman LG, Rouse MW, Brin BN. Quality of life: a review. J Am Optom Assn 1995; 66: $281-289$

14 Pain K, Dunn M, Anderson G et al. Quality of life: What does it mean in rehabilitation. Journal of Rehabilitation 1998; 64: 5 - 11

15 Schara J. Was bedeutet Lebensqualität bei Krebs. In: Aulbert E., Niederle N. (Hrsg). Die Lebensqualität des chronisch Krebskranken. Stuttgart: Thieme, 1990: 1 - 14

16 Dirhold S, Thomas W. Ein theoretischer Orientierungsrahmen zur Erforschung der Lebensqualität onkologischer Patienten. In: Petermann F. (Hrsg). Lebensqualität und chronische Krankheit. München-Deisenhofen: Dustri-Verlag, 1996: $72-96$

17 Bullinger M. Trends in der internationalen Lebensqualitätsforschung In: Petermann F. (Hrsg). Lebensqualität und chronische Krankheit. München-Deisenhofen: Dustri-Verlag, 1996,: 5-28

18 Petermann F, Krischke N, Deuchert M. Methodische Probleme bei der Erfassung der Lebensqualität. In: Petermann F, Bergmann KC. (Hrsg). Lebensqualität und Asthma. Berlin, München: Quintessenz, 1994: $29-42$

19 Lienert G, Raatz U. Testaufbau und Testanalyse. Weinheim: Psychologie Verlags Union, 1998

20 Petermann F. Asthma bronchiale. Göttingen: Hogrefe, 1999

${ }^{21}$ Gill TM, Feinstein AR. A critical appraisal of the quality of quality-oflife measurements. JAMA 1994; 272: 619-626

22 Juniper EF, Guyatt GH, Epstein RS et al. Evaluation of impairment of health related quality of life in asthma: development of a questionnaire for use in clinical trials. Thorax 1992; 47: 76-83

23 Juniper EF, Guyatt GH, Ferrie PJ et al. Measuring quality of life in asthma. Am Rev Respir Dis 1993; 147: 832-838

${ }^{24}$ Hunt SM, McKenna SP, McEwen J et al. The Nottingham Health Profile: subjective health status and medical consultations. Social Science \& Medicine - Part A, Soc Sci Med 1981; 15: 221 - 229

25 Bergner M, Bobbitt RA, Pollard WE et al. The Sickness impact profile: validation of a health status measure. Med Care 1976; 14: 57-67

${ }^{26}$ Bergner M, Bobbitt RA, Carter WB et al. The Sickness Impact Profile: development and final revision of a health status measure. Med Care 1981; 19: $787-805$

27 Ware JE Jr, Sherbourne CD. The MOS 36-item short-form health survey (SF-36). I. Conceptual framework and item selection. Med Care 1992; 30: $473-483$

${ }^{28}$ Ware JE Jr, Kosinski M, Bayliss MS et al. Comparison of methods for the scoring and statistical analysis of SF-36 health profile and summary measures: summary of results from the Medical Outcomes Study. Med Care 1995; 33: AS264-79

${ }^{29}$ Ware JE Jr. The SF-36 Health Survey. In Spilker B. (Hrsg). Quality of Life and Pharmacoeconomics in Clinical Trials. Philadelphia, New York: Lippincott-Raven, 1996: 337 - 345

${ }^{30}$ Bullinger M, Kirchberger I. SF-36 Fragebogen zum Gesundheitszustand - Handanweisung. Göttingen: Hogrefe, 1998

31 Aaronson NK, Acquadro C, Alonso J et al. International Quality of Life Assessment (IQOLA) Project. Qual Life Res 1992; 1: 349-351

32 Wagner AK, Gandek B, Aaronson NK et al. Cross-cultural comparisons of the content of SF-36 translations across 10 countries: results from the IQOLA Project. International Quality of Life Assessment. J Clin Epidemiol 1998; 51: $925-932$

33 Ware JE Jr, Kosinski M, Gandek B et al. The factor structure of the SF-36 Health Survey in 10 countries: results from the IQOLA Project. Interna- tional Quality of Life Assessment. J Clin Epidemiol 1998; 51: $1159-1165$

${ }^{34}$ Ware JE Jr, Gandek B, Kosinski M et al. The equivalence of SF-36 summary health scores estimated using standard and country-specific algorithms in 10 countries: results from the IQOLA Project. International Quality of Life Assessment. J Clin Epidemiol 1998; 51: 1167 - 1170

35 Ware JE Jr, Gandek B. Overview of the SF-36 Health Survey and the International Quality of Life Assessment (IQOLA) Project. J Clin Epidemiol 1998; 51: $903-912$

36 Zigmond AS, Snaith RP. The hospital anxiety and depression scale. Acta Psychiatr Scand 1983; 67: 361 - 370

${ }^{37}$ Herrmann C, Scholz KH, Kreuzer H. Psychologisches Sreening von Patienten einer kardiologischen Akutklinik mit einer deutschen Fassung der „Hospital Anxiety and Depression Scale“ (HAD)-Skala. PPmP Psychother Psychosom med Psychol 1991; 41: 83-92

${ }^{38}$ Herrmann C, Buss U, Breuker A et al. Beziehungen kardiologischer Befunde und standardisierter psychologischer Skalenwerte zur klinischen Symptomatik bei 3705 ergometrisch untersuchten Patienten. Z Kardiol 1994; 83: 264-272

${ }^{39}$ Herrmann C, Buss U, Lingen R et al. The screening for anxiety and depression in routine medical care. Dtsch Med Wschr 1994; 119: $1283-1286$

${ }^{40}$ Guyatt GH, Berman LB, Townsend M et al. A measure of quality of life for clinical trials in chronic lung disease. Thorax 1987; 42: $773-778$

41 Jones PW, Quirk FH, Baveystock CM. The St George's Respiratory Questionnaire. Respir Med 1991; 85 Suppl B: 25 - 31; discussion 33-7

42 Windisch W, Petermann F, Laier-Groeneveld G et al. Lebensqualität bei Heimbeatmung. Med Klin 1997; 92 Suppl 1: 95 - 100

43 Carone M, Bertolotti G, Anchisi F et al. Analysis of factors that characterize health impairment in patients with chronic respiratory failure. Quality of Life in Chronic Respiratory Failure Group. Eur Respir J 1999; 13: $1293-1300$

44 Windisch W, Petermann F, Schucher B et al. The Severe Respiratory Insufficiency (SRI) Questionnaire: A specific measure of health-related quality of life in patients receiving home mechanical ventilation. (Abstract) Eur Respir J 2001; 18, Suppl 33: 402S

45 Aaronson NK, Ahmedzai S, Bergman B et al. The European Organization for Research and Treatment of Cancer QLQ-C30: a quality-of-life instrument for use in international clinical trials in oncology. Journal of the National Cancer Institute 1993; 85: 365 - 376

${ }^{46}$ Sjoberg L, Svensson E, Persson LO. The measurement of mood. Scandinavian Journal of Psychology 1979; 20: 1 - 18

${ }^{47}$ Follick MJ, Ahern DK, Aberger EW. Development of an audiovisual taxonomy of pain behavior: reliability and discriminant validity. Health Psychology 1985; 4: 555-568

48 Pehrsson K, Olofson J, Larsson S et al. Quality of life of patients treated by home mechanical ventilation due to restrictive ventilatory disorders. Respir Med 1994; 88: 21 - 26

49 Dellborg C, Olofson J, Midgren B et al. Quality of life in patients with chronic alveolar hypoventilation. Eur Respir J 2002; 19: 113-120

50 Sullivan M, Karlsson J. The Swedish SF-36 Health Survey III. Evaluation of criterion-based validity: results from normative population. J Clin Epidemiol 1998; 51: $1105-1113$

51 Bach JR. A comparison of long-term ventilatory support alternatives from the perspective of the patient and care giver. Chest 1993; 104 : $1702-1706$

52 Hurel D, Loirat P, Saulnier F et al. Quality of life 6 months after intensive care: results of a prospective multicenter study using a generic health status scale and a satisfaction scale. Int Care Med 1997; 23: $331-337$

53 Seemungal TA, Donaldson GC, Paul EA et al. Effect of exacerbation on quality of life in patients with chronic obstructive pulmonary disease. Am J Respir Crit Care Med 1998; 157: 1418-1422

${ }^{54}$ Ambrosino N, Bruletti G, Scala V et al. Cognitive and perceived health status in patient with chronic obstructive pulmonary disease surviving acute on chronic respiratory failure: a controlled study. Int Care Med 2002; 28: 170-177

55 Elliott MW, Simonds AK, Carroll MP et al. Domiciliary nocturnal nasal intermittent positive pressure ventilation in hypercapnic respiratory failure due to chronic obstructive lung disease: effects on sleep and quality of life. Thorax 1992; 47: $342-348$

56 Perrin C, El Far Y, Vandenbos F et al. Domiciliary nasal intermittent positive pressure ventilation in severe COPD: effects on lung function and quality of life. Eur Respir J 1997; 10: 2835 - 2839 
57 Meecham Jones DJ, Paul EA, Jones PW et al. Nasal pressure support ventilation plus oxygen compared with oxygen therapy alone in hypercapnic COPD. Am J Respir Crit Care Med 1995; 152: 538 - 544

58 Sivasothy P, Smith IE, Shneerson JM. Mask intermittent positive pressure ventilation in chronic hypercapnic respiratory failure due to chronic obstructive pulmonary disease. Eur Respir J 1998; 11: $34-40$

${ }^{59}$ Lyall RA, Donaldson N, Fleming T et al. A prospective study of quality of life in ALS patients treated with noninvasive ventilation. Neurology 2001; 57: $153-156$

60 Jones PW, Bosh TK. Quality of life changes in COPD patients treated with salmeterol. Am J Respir Crit Care Med 1997; 155: 1283-1289
61 Bennett LS, Barbour C, Langford B et al. Health status in obstructive sleep apnea: relationship with sleep fragmentation and daytine sleepiness, and effects of continuous positive airway pressure treatment. Am J Respir Crit Care Med 1999; 159: 1884-1890

62 Paditz E, Zieger S, Bickhardt J et al. Lebensqualität bei Heimbeatmung im Kindes-, Jugend-, und jungen Erwachsenenalter: unterschiedliche Sichtweisen von Eltern und Kindern. Somnologie 2000; 4: 13-19

${ }^{63}$ Carone M, Ambrosino N, Bertolotti G et al. Quality of Life Evaluation and Survival Study: a 3-yr prospective multinational study on patients with chronic respiratory failure. Monaldi Arch Chest Dis 2001; 56: $17-22$

\section{Ausschreibung}

WIR-Journalistenpreis der Deutschen Lungenstiftung 2001/2002

1. Die Deutsche Lungenstiftung verleiht für die Jahre 2001 und 2002 im Rahmen der Jahrestagung der Deutschen Gesellschaft für Pneumologie erneut den WIR Journalistenpreis zur Würdigung hervorragender journalistischer Arbeiten auf dem Gesamtgebiet der Pneumologie.

2. Der Preis wird zur Erinnerung an den Lungenfacharzt Dr. Wilhelm Roloff und seine Frau Ingeborg von deren vier Söhnen gestiftet. Er soll Defizite der Medienberichterstattung über Lungenerkrankungen ausgleichen.

3. Der Preis wird an Journalistinnen und Journalisten vergeben, die sich durch einen oder mehrere Beiträge in Presse, Büchern, Hörfunk oder Fernsehen in hervorragender Weise mit aktuellen Entwicklungen der Lungenheilkunde beschäftigen. Bewerben können sich Journalisten (auch Teams) mit höchstens drei Beiträgen, die zwischen dem 1. Januar 2001 und dem 31. Dezember 2002 in deutscher Sprache veröffentlicht wurden. Zulässig sind Eigenbewerbungen sowie Empfehlungen durch Dritte.

4. Der Preis ist mit 4.000 Euro ausgestattet. Es wird ein erster Preis (2.500 Euro) sowie ein zweiter Preis (1.500 Euro) verliehen.

5. Der Vorstand der Deutschen Lungenstiftung hat eine Jury eingesetzt, die dem Vorstand aus den eingereichten Arbeiten die preiswürdigen benennt. Die Benennung erfolgt durch den Vorsitzenden der Jury in Form eines schriftlichen Gutachtens, aus dem hervorgeht, warum welcher Arbeit ein Preis zuerkannt wurde. Das Gutachten wird anlässlich der Preisverleihung durch den Vorsitzenden der Deutschen Lungenstiftung öffentlich vorgelesen. Die Preisträger werden zur Verleihung nach München eingeladen.
6. Die Arbeiten müssen bis zum 15. 1. 2003 (Datum des Poststempels) beim Vorsitzenden der Deutschen Lungenstiftung, Herrn Prof. Dr. med. Helmut Fabel, unter folgender Anschrift eingereicht werden:

\section{Deutsche Lungenstiftung e. $V$. \\ Herrn Prof. Dr. med. Helmut Fabel \\ Herrenhäuser Kirchweg 5, 3. OG \\ 30165 Hannover \\ Tel.: 0511/2155110, Fax: 0511/2155113 \\ E-mail: Deutsche.Lungenstiftung@t-online.de www.lungenstiftung.de}

7. Die Bewerber werden gebeten, den Arbeiten neben Veröffentlichungs- bzw. Sendedatum, Angaben zu Namen, Adresse, Lebenslauf mit Ausbildung und journalistischem Werdegang sowie zur derzeitigen Tätigkeit anzufügen.

8. Für das Thema der einzureichenden Beiträge gibt es keine eng begrenzten Vorgaben. Erwünscht sind Arbeiten, die sich sowohl mit den gravierenden, aber weithin unterschätzten Problemen der Lungenheilkunde in Forschung und Praxis befassen, als auch solche, die sich mit Einzelfragen beschäftigen, z. B. zu einzelnen Lungenerkrankungen. Erwünscht sind ebenso Arbeiten zur Früherkennung und Prävention von Lungenkrankheiten, zum Nichtraucherschutz, zu NichtraucherInitiativen, zur Strategie der Tabakindustrie und zur medizinischen Bedeutung des Werbeverbots, das innerhalb der EU für Tabakwaren vorgesehen ist.

9. Mit der Vergabe der Preise erhält die Deutsche Lungenstiftung das Recht, die ausgezeichneten Beiträge in der Mitgliederzeitschrift „Lunge, Luft und Leben“ zu veröffentlichen. Die eingesandten Arbeiten werden nur auf Anfrage an die Bewerber zurückgesendet; eine Haftung kann nicht übernommen werden. 